\title{
Caracterización de un Sistema GPS RTK de Bajo Costo
}

\section{Characterization of a low cost GPS RTK system}

\author{
Marcelo Pistarelli \\ CIFASIS, Centro Internacional Franco Argentino de Ciencias de la Información y de Sistemas \\ (CONICET-UNR) - Argentina \\ mpistare@fceia.unr.edu.ar
}

\section{Taihú Pire}

CIFASIS, Centro Internacional Franco Argentino de Ciencias de la Información y de Sistemas (CONICET-UNR) - Argentina

pire@cifasis-conicet.gov.ar

\section{Ernesto Kofman}

CIFASIS, Centro Internacional Franco Argentino de Ciencias de la Información y de Sistemas (CONICET-UNR) - Argentina

kofman@cifasis-conicet.gov.ar

\section{Resumen}

En las últimas décadas la comunidad robótica ha adoptado cada vez más el uso de sensores GPS-RTK para estimar de forma precisa la localización de robots móviles en ambientes exteriores. En este trabajo se presenta la caracterización de un sistema GPS-RTK de bajo costo. A partir del montaje de las unidades base y móvil se adquirieron datos de posición corregidos en tiempo real por el mismo sistema RTK bajo diferentes disposiciones físicas, velocidades de actualización y condiciones ambientales presentándose posteriormente un estudio estadístico de los resultados obtenidos.

Palabras claves: GPS, RTK, Bajo Costo, GLONASS, RTKLib, REACH. 


\begin{abstract}
In recent decades, the robotic community has increasingly adopted the use of GPS-RTK sensors to accurately estimate the location of mobile robots in outdoor environments. In this work, the characterization of a low-cost GPS-RTK system is presented. From the assembly of the base and mobile units, corrected position data was acquired in real time by the same RTK system under different physical dispositions, update rates and environmental conditions, and a statistical study of the results obtained was subsequently presented.
\end{abstract}

keywords: GPS, RTK, Low Cost, GLONASS, RTKLib, REACH.

\title{
INTRODUCCIÓN
}

El uso de un Sistema de Posicionamiento Global (GPS) o un sistema global de navegación por satélite (GNSS) para tareas de robótica móvil en ambientes exteriores resulta de gran importancia, dado que provee una estimación de la posición del robot en un sistema de coordenadas global independientemente del entorno que lo rodea. Sin embargo, debido a la baja precisión que presentan los GPS estándares, su medición de posicionamiento suele ser insuficiente para llevar adelante la navegación de un robot, teniendo que fusionar dicha estimación con otros sistemas de medición montados sobre el robot como cámaras, láser, unidades de medición inercial, entre otros.

En las últimas décadas, ha surgido el sistema de Navegación Cinética Satelital en Tiempo Real (RTK, por las siglas en inglés de Real Time Kinematic) que permite estimar de manera submétrica en tiempo real la posición de un robot. Los mismos constan de una estación base receptora de señal GPS que transmite mediante un enlace de datos inalámbrico la señal de corrección a una o varias unidades móviles. A partir de la combinación de la señal de corrección con la señal GPS recibida, cada unidad móvil realiza el cálculo en tiempo real de la posición relativa con respecto a la base con precisión del orden del centímetro. Estando la precisión de la posición absoluta limitada por la de la estación base. De esta manera, los GPS-RTK brindan una estimación de posición suficientemente precisa para la mayoría de las aplicaciones de robótica móvil, como por ejemplo, agricultura de precisión [1], [2], [3], [4], monitoreo de mareas [5] y predicción de tsunamies [6].

Recientemente, han surgido sistemas GPS-RTK de bajo costo, ampliando el acceso de dicha tecnología, y haciendo más redituables a las aplicaciones robóticas comerciales que los implementan. A diferencia de los sistemas de uso profesional, los GPS-RTK de bajo costo presentan diversas limitaciones. ofrecen velocidades de actualización entre 10 y 25 veces menores a los de su contra parte, reduciendo el rango de aplicaciones dinámicas posibles. Además, poseen receptores de señal GPS que operan en una sola banda de frecuencias (L1), a diferencia de los de mayor costo que trabajan con 2 bandas (L1 y L2) obteniendo mayor precisión. Por otro lado, los sistemas GPS-RTK de bajo costo del mercado se encuentran en forma de placas o módulos electrónicos que carecen del equipo de enlace de radio integrado, requiriendo tiempo de montaje y configuración. También, otra limitación que presentan es la calibración de las lecturas de posición obtenidas una vez realizado el montaje del sistema final. Más allá de esta serie de desventajas o limitaciones, resulta importante evaluar la precisión y robustez de un sistema GPS-RTK de bajo costo con el fin de poder ser utilizado para asistir a métodos de navegación para robots móviles y como groundtruth para evaluar 
sistemas de localización basados en otros sensores como cámaras o lásers.

En este trabajo, se realiza una completa caracterización de un GPS-RTK de bajo costo. En particular, se trabaja sobre el GPS-RTK Reach de la empresa Emlid. Se analiza la precisión variando la frecuencia de muestreo. Asimismo, se detalla la puesta a punto tanto del transmisor en la estación base como del receptor en la unidad móvil, importante para su fácil implementación.

El trabajo está estructurado de la siguiente manera: en la sección II se comenta el trabajo relacionado. En la sección III se caracteriza el GPS RTK de bajo costo y se detalla la configuración utilizada para el mismo. En la sección IV se muestran los experimentos que demuestran la precisión alcanzada por el GPR-RTK. Finalmente, en la sección V se comentan las conclusiones que derivan del presente trabajo.

\section{TRABAJO RELACIONADO}

Existen varios trabajos que realizan estudios sobre la precisión de los sistemas GPS-RTK. Feng y Wang [7] estudian cómo el sistema GPS-RTK con múltiples constelaciones de satélites funciona utilizando señales GPS y mediciones de Galileo virtuales de doble frecuencia. Más tarde los mismos autores en [8] definen y detallan características a considerar sobre los parámetros de Resolución de Ambigüedad (AR, por las siglas en inglés de Ambiguity Resolution) y de estimación de posicionamiento para tener una mayor comprensión sobre la performance de los sistemas GPS-RTK. Por otro lado, en [9] y [10] se propone la utilización de información inercial para mejorar precisión ante pérdidas de señal GPS. Logrando durante este lapso precisión del orden del decímetro.

El trabajo realizado en [11] presenta la evaluación del sistema GPS-RTK de bajo costo NVO8C-CSM con el fin de ser utilizados para aplicaciones de agricultura y medio ambiente. Para su evaluación se realizaron experimentos con una configuración estática (la unidad móvil permanece en un lugar fijo) y una configuración dinámica (la unidad móvil se desplaza libremente). Los autores reportan una desviación de $5 \mathrm{~mm}$ para cuando la unidad móvil permanece quieta en una posición y de $12 \mathrm{~mm}$ cuando ésta se mueve libremente.

En [12] se evalúa también el GPS-RTK NVO8C-CSM pero con vistas de ser aplicado para vehículos no tripulados que trabajen en la superficie (Unmanned Surface Vehicle, USV). En particular, los autores proponen implementar dicho sistema en robots marítimos. El trabajo muestra que la precisión obtenida es suficiente como para emplear dicho sistema de localización en tareas tales como Búsqueda y Rescate (Search \& Rescue, S\&R) de personas en el mar.

En [13] se hace una evaluación de dos sistemas GPS-RTK de bajo costo (Skytraq NS-RAW y Ublox M8N) analizando la performance de los mismos bajo una configuración estática y una dinámica. Además, para ambas configuraciones se comparan los resultados obtenidos con un sistema GPS-RTK de alta gama de dos bandas (L1 y L2). Como resultado de la comparación se muestra que los GPS-RTK de bajo costo tienen una precisión comparable con la de un GPS-RTK de alta gama.

Siguiendo la línea de los trabajos antes mencionados, en este trabajo se propone complementar la evaluación los GPS-RTK de bajo costo mediante su caracterización bajo distintas condiciones climáticas y distintas frecuencia de muestreo de los receptores involucrados. Además, se aborda la caracterización desde un punto de vista para su aplicación en el campo de la robótica móvil. 


\section{CARACTERIZACIÓN DEL GPS-RTK}

A. Principio de funcionamiento del sistema de posicionamiento satelital

El sistema GPS consta de una constelación de al menos 24 satélites operativos que orbitan la tierra a $20.200 \mathrm{Km}$ de altura recorriéndola dos veces por día. Se encuentra diseñado de forma que se pueda contar con un mínimo de 4 satélites visibles por encima de un ángulo de elevación de $15^{\circ}$ en cualquier punto de la superficie terrestre. Cada satélite GPS lleva a bordo relojes atómicos muy precisos que operan en una frecuencia fundamental de $10.23 \mathrm{MHz}$, la cual se emplea para generar las señales transmitidas por el satélite.

Los satélites transmiten constantemente códigos pseudoaleatorios en dos ondas portadoras. Estas ondas portadoras se encuentran en la banda L. La portadora Ll es transmitida a 1575.42 MHz y la portadora L2 es transmitida a $1227.60 \mathrm{MHz}$. La portadora Ll es modulada por dos códigos. El Código C/A o Código de Adquisición Gruesa modula a 1.023MHz y el código $\mathrm{P}$ o Código de Precisión modula a 10.23MHz. L2 es modulada por el código P únicamente.

Los receptores GPS utilizan los diferentes códigos pseudoaleatorios para distinguir los satélites. Los códigos también son empleados como base para realizar las mediciones de pseudodistancia y a partir de ahí, calcular una posición. La posición del receptor se podrá determinar al intersecar tres esferas imaginarias, obtenidas a partir de la comparación del código generado por el receptor GPS y el recibido como puede verse en la Fig. 1. Para la corrección del error introducido por el reloj del receptor se incorpora un cuarto satélite en el cálculo.

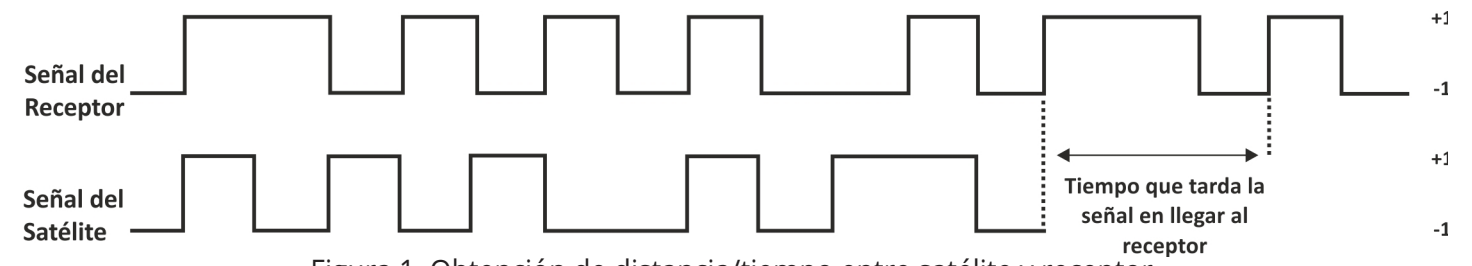

Figura 1. Obtención de distancia/tiempo entre satélite y receptor.

B. Fuentes de error del sistema GPS

El sistema GPS está sometido a diferentes fuentes de error:

Retrasos ionosféricos y atmosféricos. Al atravesar la señal GPS la ionosfera, su velocidad puede disminuir. Estos retrasos pueden introducir error en el cálculo de la distancia. La ionosfera no introduce un retraso constante en la señal ya que influyen factores como la elevación del satélite y las variaciones de densidad ocasionadas por el Sol.

Efecto Multitrayectoria. Se presenta cuando el receptor está ubicado cerca de una superficie reflectora como un lago o un edificio. La señal del satélite no viaja directamente a la antena, sino que llega al objeto cercano y luego es reflejada a la antena, provocando una medición falsa. Este tipo de errores son reducidos utilizando antenas GPS especiales que incorporan un plano de tierra el cual evita que las señales con poca elevación lleguen a la antena.

Dilución de la Precisión. Es una medida de la fortaleza de la geometría de los satélites y está relacionada con la distancia entre éstos y su posición en el cielo. El DOP puede incrementar el efecto del error en la medición de distancia a los satélites. Para minimizar el efecto de la DOP se debe observar tantos satélites como sean posibles. Sin embargo, las señales de satélites con poca elevación son más propensas a ser afectadas por los retrasos ionosféricos, atmosféricos y el efecto multitrayectoria. 


\section{Sistema RTK}

Los últimos estudios realizados por la Administración Federal de Aviación de los Estados Unidos sobre la performance del sistema de posicionamiento global GPS indican que el error en la posición de un receptor convencional de una única frecuencia en buenas condiciones de recepción es en el 95 \% de las veces inferior a 1.891 metros. Ésto se debe a las numerosas fuentes de error detalladas anteriormente.

A diferencia de los sistemas GPS estándar donde no es posible atenuar el efecto de las fuentes de error por tratarse de un sistema conformado por un único receptor, los sistemas de posicionamiento GPS-RTK al estar compuestos por dos o más receptores cercanos entre si son afectados por las mismas fuentes de error, lo que permite la utilización de algoritmos de corrección para lograr una mejora en la precisión. La configuración de un sistema GPSRTK está dada por un receptor de referencia (estación base) que está siempre ubicado en un punto fijo y uno o varios receptores móviles (unidades móviles). Ambos tipos de receptores deben observar la misma constelación de satélites al mismo tiempo estando ubicados a una distancia inferior a los $20 \mathrm{Km}$. De esta forma, las señales recibidas por los receptores son afectadas por las mismas fuentes de error, y puesto que la posición de la base es conocida es posible realizar correcciones logrando precisión submétrica. En la Figura 2 se ilustran los principales componentes del sistema GPS-RTK.

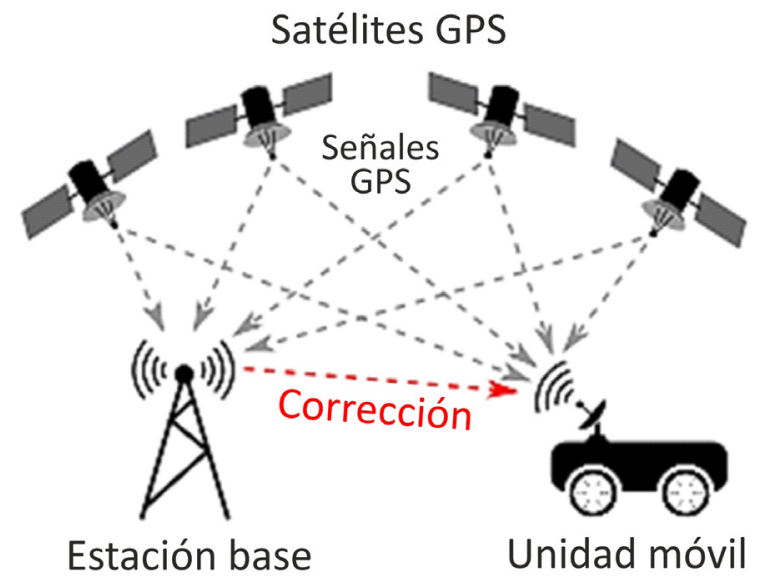

Figura 2. Sistema GPS-RTK.

Las correcciones se generan a partir de la medición basada en la fase portadora, ya que puede proporcionar una medida hacia el satélite mucho más precisa que la que se consigue utilizando el código C/A o el código P. La onda portadora de L1 tiene una longitud de $19.4 \mathrm{~cm}$. A partir de la medición del número de longitudes de onda (completas y fraccionarias) que existen entre el satélite y el receptor se obtiene una distancia muy precisa al satélite. La determinación de la cantidad de longitudes de onda (Carrier Phase Ambiguity Fixing) representa un problema debido a la existencia de muchas posibles de longitudes de onda enteras para cada satélite, siendo la solución ambigua. Ésto se resuelve mediante procesos estadísticos determinando la solución más probable.

Para la determinación de la cantidad de longitudes de onda, se parte de la obtención de una posición aproximada (estado Float) cuyo error define un círculo dentro del cual se encuentra la posición exacta. Los frentes de onda de un satélite al ser intersecados con el círculo, forman un conjunto de líneas. Siendo uno de los puntos de estas líneas la solución 
correcta.

Mediante la incorporación de más satélites al cálculo se van obteniendo nuevos conjuntos se líneas intersecados, reduciendo la cantidad de posibles soluciones correctas. A medida que transcurre el tiempo los satélites cambian de posición generando nuevos conjuntos de soluciones posibles que a partir de un análisis estadístico permiten obtener la posición correcta (estado Fix). Este proceso se ilustra en la Figura 3. Logrando una precisión relativa a la base del orden del centímetro, estando la precisión de la posición absoluta limitada a la de la base.

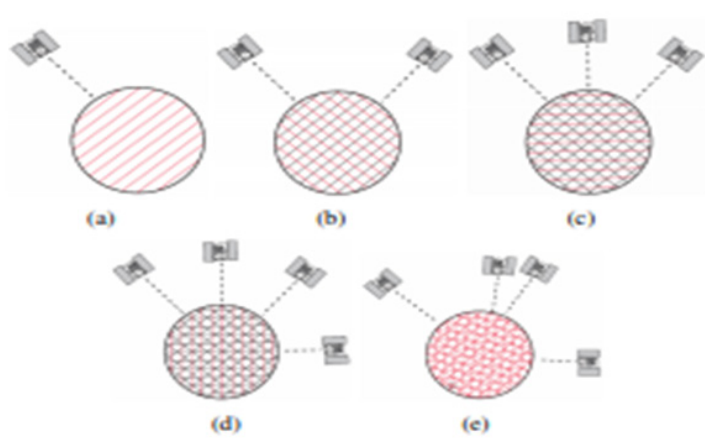

Figura 3. a: Determinación de posición aproximada estado Float). b,c,d: Incorporación de satélites adicionales para la reducción del conjunto de posibles posiciones (estado Float). e: Combinación de los conjuntos de posibles soluciones a lo largo del tiempo para la determinación de la posición correcta (estado Fix).

D. GPS-RTK de bajo costo

Para este trabajo se seleccionó el sistema GPS-RTK Reach de la empresa Emlid por su bajo costo, capacidades de interconexión con otros dispositivos electrónicos, compatibilidad con unidades base RTK profesionales y la posibilidad de monitorear en tiempo real el estado de recepción de señal GPS de la unidad móvil y base.

Está constituido por dos unidades de procesamiento idénticas configuradas para comportarse una como base y otra como unidad móvil. A su vez, cada unidad de procesamiento está compuesta por un módulo GPS u-blox NEO-M8T de uso profesional conectado a un módulo de procesamiento INTEL Edison donde se ejecuta el Firmware de posicionamiento y corrección en tiempo real.

Para la realización de las correcciones el sistema hace uso del paquete de código abierto RTKLIB [14]. Está compuesto de múltiples librerías escritas en lenguaje C que proveen las principales funciones de navegación, algoritmos de posicionamiento relativo basados en portadora para posicionamiento RTK con resolución de ambigüedad de entero, entre otras funcionalidades. Permitiendo que las correcciones puedan ser realizadas en tiempo real o post-proceso. También, soporta diversos GNSS: GPS, GLONASS, GALILEU, QZSS, BeiDou y SBAS.

El sistema GPS-RTK Reach soporta diferentes protocolos de capa de aplicación para el intercambio de los mensajes de corrección entre los que se destaca RTCM (The Radio

Technical Commission for Maritime Services). La utilización de RTCM otorga interoperabilidad al sistema, permitiendo que una unidad de posicionamiento móvil Reach reciba información de corrección en tiempo real de una base desarrollada por otro fabricante.

E. Configuración de hardware 
Teniendo como premisa el desarrollo flexible, modular y multiplataforma se montaron la estación base y unidad móvil en el interior de cajas plásticas estancas. En particular, la unidad móvil fue concebida con el fin de que pueda ser montada fácilmente en un robot móvil terrestre.

La estación base está compuesta por un módulo Reach conectado a una antena Tallysman TW4721 con protección IP67. La misma se encuentra montada sobre un plano de tierra de 322 x $247 \mathrm{~mm}$ que supera ampliamente el sugerido por el fabricante del GPS-RTK de $100 \mathrm{x}$ $100 \mathrm{~mm}$, otorgándole un rechazo superior ante señales provenientes de rebotes en las estructuras cercanas (señales multiruta).

Para energizar ambos sistemas se optó por la incorporación de un módulo alimentado por cuatro celdas de litio recargables con una capacidad total de $10.400 \mathrm{mAh}$ que integra el sistema de carga y salida regulada switching de tensión de $5 \mathrm{~V}$ en un mismo contenedor.

\section{EXPERIMENTOS}

Se realizaron experimentaciones estáticas y dinámicas adquiriendo datos de posición de la unidad móvil bajo diferentes condiciones ambientales con el fin de estudiar la precisión y robustez del sistema de bajo costo seleccionado.

En cada uno de los experimentos se fijaron los mismos grupos de satélites capturados GPS/GPS-GLONASS y frecuencia de actualización tanto en la estación base como en la unidad móvil.

A. Experimentación I Estando el cielo totalmente cubierto por nubes, se montó la estación base sobre un trípode libre de la presencia de obstáculos en la terraza del instituto CIFASIS. A 5 metros de distancia se ubicó la unidad móvil y se realizó la adquisición de datos de posición durante 15 minutos. El muestreo se realizó a $1 \mathrm{~Hz}$ y $5 \mathrm{~Hz}$, incorporando en el cálculo de la posición las señales los sistemas de satélites GPS y GLONASS. Posteriormente se repitió el experimento sólo con los satélites GPS a $1 \mathrm{~Hz}, 5 \mathrm{~Hz}, 10 \mathrm{~Hz}$ y 14Hz. En la Tabla I se puede ver la proporción entre el tiempo en Fix y Float para las diferentes frecuencias de actualización. Se observa que se obtienen mejores resultados, es decir mayor tiempo en estado Fix, cuando se reciben señales de las dos familias soportadas por el receptor, disponibles en el territorio Argentino (GPS y GLONASS). Esto se debe a que al aumentar cantidad de satélites en la línea de vista de los receptores GPS-RTK, mayor es la cantidad de señales que pueden ser procesadas por los receptores, aumentando las posibilidades de alcanzar el estado Fix. En la Fig. 4 se muestran, para la mejor configuración (GPS- GLONASS-5Hz), los resultados obtenidos de posición en las direcciones E-0, N-S y altitud. Se observa que inicialmente el sistema arroja

\begin{tabular}{|c|c|c|c|}
\hline Satelites & Fix $(\%)$ & Float $(\%)$ & Frec $(\mathrm{Hz})$ \\
\hline GPS - GLONASS & 54.67 & 45.33 & 1 \\
\hline GPS - GLONASS & 27.11 & 72.89 & 5 \\
\hline GPS & 43.63 & 56.37 & 1 \\
\hline GPS & 1.11 & 98.89 & 5 \\
\hline GPS & 11.62 & 88.38 & 10 \\
\hline GPS & 7.34 & 92.66 & 14 \\
\hline
\end{tabular}

Tabla 1:relación entre estados fix y float con el cielo totalmente cubierto para las diferentes configuraciones estudiadas. 
valores de posición muy lejanos a los reales, a medida que realiza las sucesivas correcciones se aproxima a la posición correcta. Demorando en este caso alrededor de 6 minutos en lograrlo, manteniendo el estado Fix por 4 minutos. Posteriormente el sistema cambió a estado Float arrojando valores a $5 \mathrm{~m}$ de la posición real, realizando una serie de correcciones que arrojaron sucesivos estados fix de corta duración erróneos. Estos valores pueden deberse a las alteraciones en la atmósfera presentes en el momento de la experimentación con el cielo totalmente cubierto.

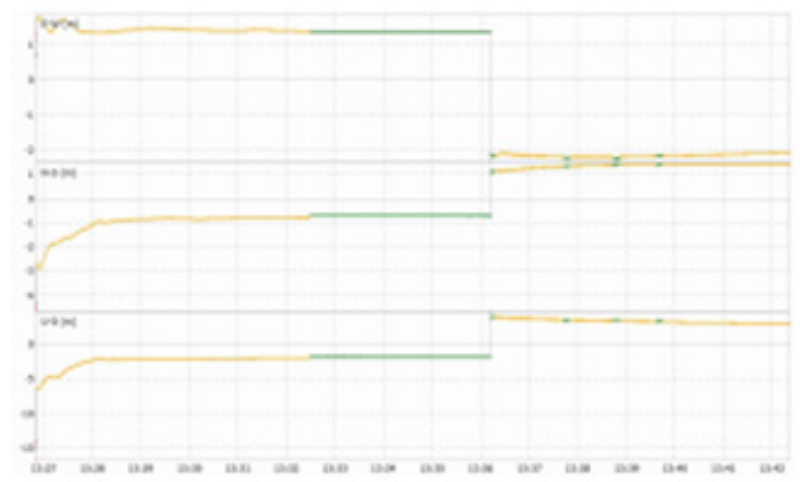

Fig. 4

La Fig. 4 muestra que el sistema puede indicar valores de posición distantes de la posición real aun estando en el estado Fix. Si bien es posible eliminar o atenuar el efecto de estos outlier de posición con diferentes técnicas, se ha estudiado la relación que tienen con respecto al nivel de resolución de ambigüedad (AR) característico de los sistemas RTK. El umbral AR por defecto para la determinación del estado Fix utilizado por el sistema de bajo costo es de 3,2, en la Tabla II se presentan los valores de error medio y desviación estandard para diferentes valores de AR. Se puede observar que existe una relación directa entre el umbral de AR elegido y la mejora en la precisión obtenida. También se puede observar que un aumento en la frecuencia de muestreo para un mismo número de satélites visibles implica una degradación de la precisión.

B. Experimentación II

Con el cielo totalmente despejado, se dispuso la misma configuración física que en el experimento anterior, y se realizaron las mismas mediciones en las diferentes frecuencias de actualización disponibles.

\begin{tabular}{|c|c|c|c|c|c|c|c|c|c|c|c|}
\hline \multirow{2}{*}{ Satelites } & \multirow{2}{*}{ Frec $(\mathrm{Hz})$} & \multicolumn{2}{|c|}{$\mathrm{AR}>3.2$} & \multicolumn{2}{c|}{$\mathrm{AR}>200$} & \multicolumn{2}{c|}{ AR $>400$} & \multicolumn{2}{c|}{ AR $>600$} & \multicolumn{2}{c|}{ AR $>800$} \\
\cline { 3 - 13 } & & $\mathrm{ti}$ & $\sigma$ & $\mathrm{ti}$ & $\sigma$ & $\mathrm{ti}$ & $\sigma$ & $\mathrm{ti}$ & $\sigma$ & $\mathrm{ti}$ & $\sigma$ \\
\hline GPS - GLONASS & 1 & 4.24 & 2.83 & 3.73 & 2.72 & 3.04 & 2.43 & 1.99 & 1.76 & 1.42 & 1.50 \\
\hline GPS - GLONASS & 5 & 59.66 & 206.13 & -3.54 & 71.06 & -6.29 & 21.91 & -5.86 & 23.98 & -6.38 & 2.20 \\
\hline GPS & 1 & 13.65 & 256.52 & -0.53 & 1.56 & -0.69 & 1.47 & -1.02 & 1.17 & -1.19 & 1.19 \\
\hline GPS & 5 & $2.10 \mathrm{e}+05$ & $5.56 \mathrm{e}+05$ & - & - & - & - & - & - & - & - \\
\hline GPS & 10 & $2.35 \mathrm{e}+04$ & $1.99 \mathrm{e}+05$ & -48.46 & $2.66 \mathrm{e}+03$ & -24.42 & $2.92 \mathrm{e}+03$ & -167.67 & 1.61 & -167.78 & 1.63 \\
\hline GPS & 14 & -0.19 & 15.52 & -0.73 & 1.55 & -1.55 & 1.27 & -1.62 & 1.38 & - & - \\
\hline
\end{tabular}

Tabla 2. Valores de error expresados en milímetros de media y desviación estándar 
con cielo cubierto para las distintas configuraciones analizadas.

En la Tabla III se muestra la proporción entre el tiempo en Fix y Float para las diferentes frecuencias de actualización. Vemos que existe una relación directa entre la mejora en las condiciones climáticas y la proporción de posiciones obtenidas con estado Fix, manteniendo el comportamiento observado en la experimentación I, donde se observó que cuando se reciben señales de las dos familias soportadas por el receptor (GPS y GLONASS) aumentan las posibilidades de alcanzar el estado Fix.

\begin{tabular}{|c|c|c|c|}
\hline Satelites & Fix $(\%)$ & Float $(\%)$ & Frec $(\mathrm{Hz})$ \\
\hline GPS - GLONASS & 85.87 & 14.13 & 1 \\
\hline GPS - GLONASS & 62.88 & 37.12 & 5 \\
\hline GPS & 0 & 100 & 1 \\
\hline GPS & 15.36 & 84.64 & 5 \\
\hline GPS & 71.75 & 28.25 & 10 \\
\hline GPS & 63.91 & 36.09 & 14 \\
\hline
\end{tabular}

Tabla 3: relación entre estados fix y float con el cielo totalmente despejado para las diferentes configuraciones estudiadas.

En la Fig. 5 se muestran, para la misma configuración expuesta en la experimentación I, los resultados obtenidos de posición en las direcciones E-0, N-S y altitud. Vemos que inicialmente el sistema arroja valores de posición lejanos $(1.9 \mathrm{~m})$ a los reales, a medida que realiza las sucesivas correcciones se aproxima a la posición correcta. Demorando en este caso alrededor de 6 minutos en lograrlo, manteniendo el estado Fix por 9 minutos. El sistema mantuvo el estado Fix hasta que se detuvo la experimentación manualmente por haber cumplido el tiempo estipulado de 15 minutos de muestreo. A diferencia de la experimentación I, no arrojó estados Fix intermitentes con valores de posición erróneos. Comprobando de esta manera que las condiciones meteorológicas tienen influencia en la estabilidad de los resultados obtenidos.

En la Tabla IV se presentan los valores de error medio y desviación estándar para los diferentes valores de AR utilizados en la experimentación anterior. Se puede observar poca variación del error medio con respecto a los diferentes valores del umbral de AR. Esto se debe a la mejora generalizada de la calidad de los resultados obtenidos asociada a la mejora en las condiciones climáticas respecto del experimento I. No se observa degradación de la precisión concluyente con el aumento de la frecuencia de muestreo. 


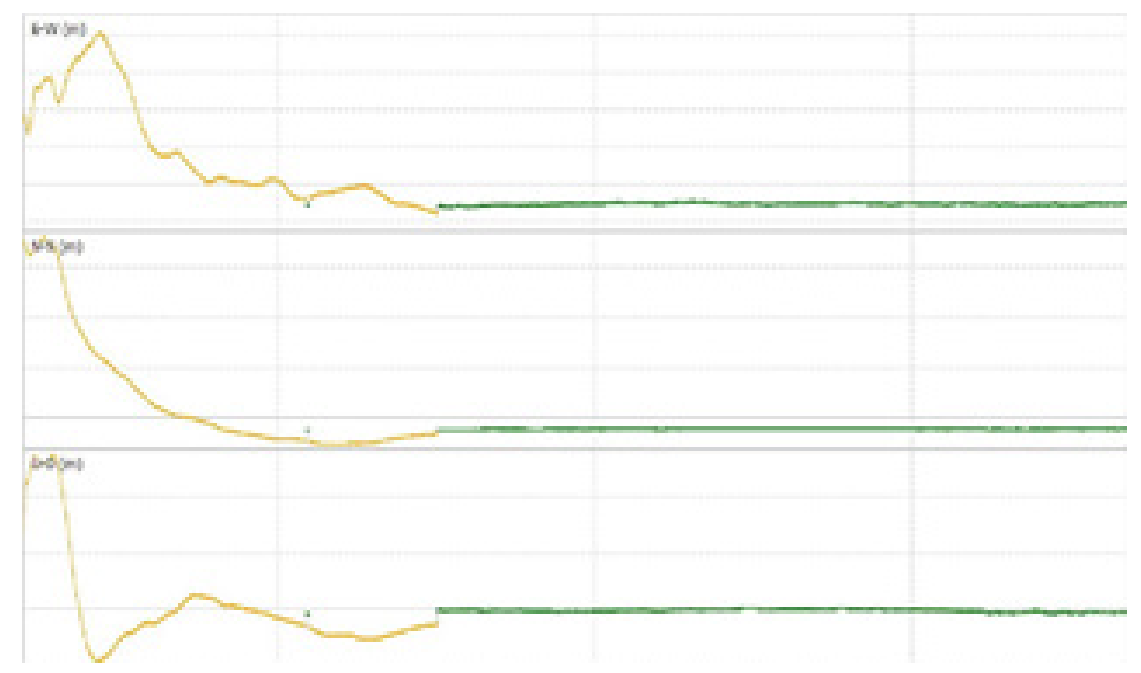

Figura 5. Estados Float (naranja) y Fix (verde) en direcciones E-0 (superior), $\mathrm{N}-\mathrm{S}$ (centro) y altitud (inferior) con cielo despejado.

\section{Experimentación III}

Se realizó el ensayo dinámico en las mismas condiciones meteorológicas que en la experimentación II. Con cielo despejado y el sistema RTK recibiendo señales de los satélites GPS y GLONASS con una velocidad de actualización de $1 \mathrm{~Hz}$ en estado FIX. Como se puede ver en la Fig. 6 se siguió repetidas veces una trayectoria circular de aproximadamente $1.5 \mathrm{~m}$ de radio a velocidad constante con el fin de analizar si el sistema es capaz de seguirla.

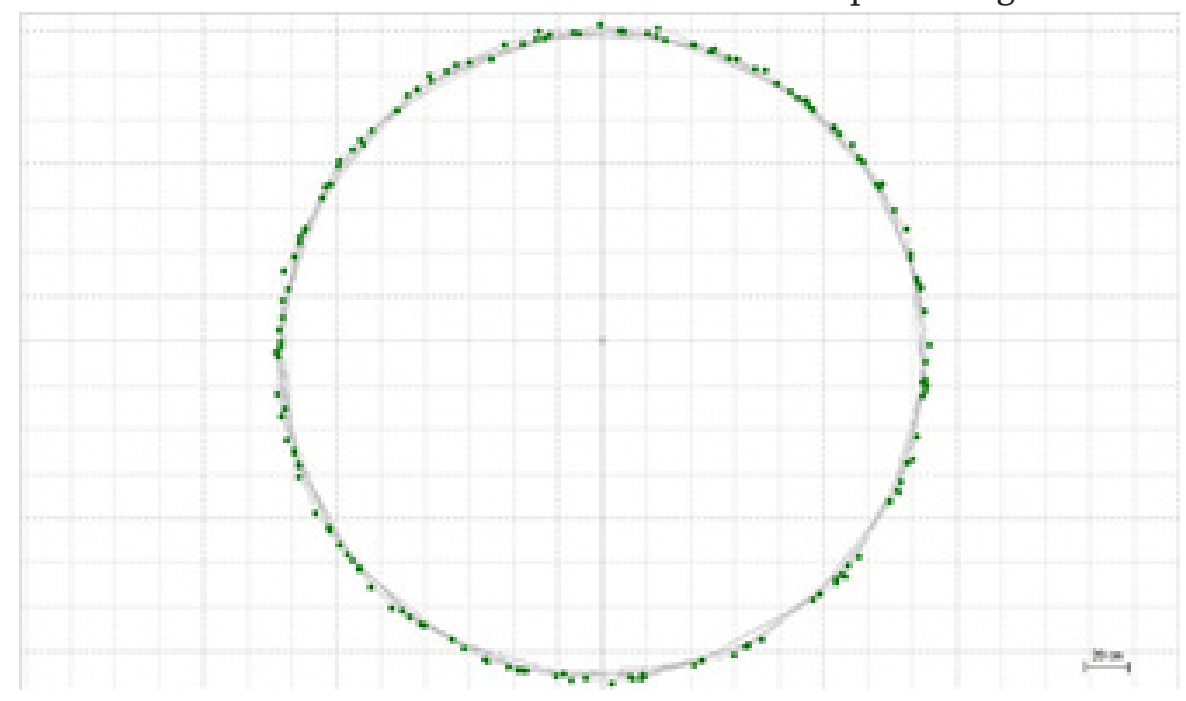

Figura 6. Posición en estado Fix durante el ensayo dinámico. 20mm/división. 
Los resultados obtenidos representan de manera correcta la trayectoria circular. Esto se puede comprobar a partir del análisis de la Fig. 7 donde se muestra la variación de

\begin{tabular}{|c|c|c|c|c|c|c|c|c|c|c|c|}
\hline \multirow{2}{*}{ Satélites } & \multirow{2}{*}{ Free $(\mathrm{Hz})$} & \multicolumn{2}{|c|}{$\mathrm{AR}>3.2$} & \multicolumn{2}{c|}{$\mathrm{AR}>200$} & \multicolumn{2}{c|}{$\mathrm{AR}>400$} & \multicolumn{2}{|c|}{$\mathrm{AR}>600$} & \multicolumn{2}{|c|}{$\mathrm{AR}>800$} \\
\cline { 3 - 13 } & & $\mathrm{ti}$ & $\sigma^{-}$ & $\mathrm{ti}$ & $\sigma^{-}$ & $\mathrm{ti}$ & $\sigma^{-}$ & $\mathrm{ti}$ & $\sigma^{-}$ & $\mathrm{ti}$ & $\sigma$ \\
\hline GPS - GLONASS & 1 & -1.30 & 3.16 & -0.93 & 2.92 & -1.22 & 3.03 & -2.23 & 3.01 & -2.69 & 2.83 \\
\hline GPS - GLONASS & 5 & -3.01 & 2.59 & -2.79 & 2.41 & -2.74 & 2.36 & -2.67 & 2.27 & -2.64 & 2.16 \\
\hline GPS & 1 & - & - & - & - & - & - & - & - & - & - \\
\hline GPS & 5 & 1.36 & 1.95 & 0.77 & 1.37 & 0.75 & 1.39 & - & - & - & - \\
\hline GPS & 10 & -0.38 & 1.85 & -0.40 & 1.86 & -0.39 & 1.89 & -0.19 & 1.90 & -0.09 & 2.06 \\
\hline GPS & 14 & -6.24 & 3.15 & -6.46 & 3.18 & -6.89 & 2.89 & -7.55 & 2.20 & -7.74 & 2.05 \\
\hline
\end{tabular}

Tabla 4: Valores de error expresados en milímetros de media y desviación estándar con cielo despejado para las distintas configuraciones analizadas.

la posición en las direcciones E-0 y N-S a lo largo del tiempo, apreciándose dos sinusoidales desfasadas 90 grados, característica de una trayectoria circular.

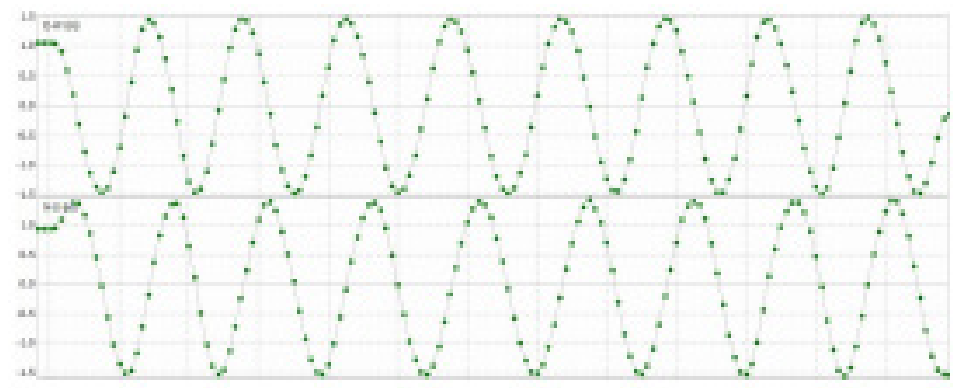

Figura 7. Variación de posición en estado Fix durante el ensayo dinámico en direcciones E-0 (superior) y N-S (inferior).

\section{CONCLUSIONES Y TRABAJO FUTURO}

En este trabajo se presenta una completa caracterización de un GPS-RTK de bajo costo. Para abordar dicha caracterización se llevaron acabo experimentos que permitieron determinar tanto la precisión como la robustez ante variaciones en la señal del GPS-RTK. Los resultados obtenidos muestran que el sistema GPS-RTK en buenas condiciones ambientales (cielo despejado) alcanza una precisión de $5 \mathrm{~mm}$ cuando se encuentra en un estado Fix, con una frecuencia de muestreo de $5 \mathrm{~Hz}$. Estando el cielo totalmente cubierto el sistema tuvo dificultades para lograr el estado Fix, y en consecuencia los valores de posición obtenidos fueron de menor precisión.

El sistema evaluado, bajo buenas condiciones atmosféricas, puede ser utilizado como ground-truth para la evaluación de métodos de localización en entornos exteriores.

Se espera complementar el presente trabajo a partir de la adquisición de un sistema GPSRTK de altas prestaciones que permitirá la realización de ensayos dinámicos a diferentes velocidades de actualización y condiciones ambientales, así como también experimentaciones 
estáticas a mayores distancias base-unidad móvil que las impuestas por las limitaciones de la metodología utilizada.

\section{AGRADECIMIENTOS}

Este trabajo se encuentra en el marco del proyecto Desarrollo de un Robot Desmalezador de la Unidad Ejecutora CIFASIS, Centro Internacional Franco Argentino de Ciencias de la Información y de Sistemas (CONICET-UNR). Además, los autores agradecen a Franco Romano, David Fattore y Lucas

Manavella por el soporte técnico brindado en la realización de las diferentes experimentaciones. 


\section{REFERENCIAS}

[1] H. Wang and N. Noguchi, "Autonomous maneuvers of a robotic tractor for farming," in 2016 IEEE/SICE International Symposium on System Integration (SII), December 2016, pp. 592-597.

[2] P. Dabove and A. M. Manzino, "GPS mass-market receivers for precise farming," in 2014 IEEE/ION Position, Location and Navigation Sympo $\operatorname{sium~-~PLANS~2014,~May~2014,~pp.~}$ 472-477.

[3] L. Cordesses, C. Cariou, and M. Berducat, "Combine Harvester Control Using Real Time Kinematic GPS," Precision Agriculture, vol. 2, no. 2, pp. 147-161, October 2000. [Online]. Available: https://doi.org/10.1023/A:1011473630247

[4] S. M. Pedersen, S. Fountas, H. llave, and B. S. Blackmore, "Agricultural robotssystem analysis and economic feasibility," Precision Agriculture, vol. 7, no. 4, pp. 295-308, September 2006. [Online]. Available: https://doi.org/10.1007/s11119-006-9014-9

[5] S. H. M. Ngagipar and O. M. Yusof, "RTK GPS water level measurement on dynamic sea surface," in 2011 IEEE Control and System Graduate Research Colloquium, June 2011, pp. $82-85$.

[6] Y. Ohta, T. Kobayashi, H. Tsushima, S. Miura, R. Hino, T. Takasu, H. Fujimoto, T. linuma, K. Tachibana, T. Demachi, T. Sato, M. Ohzono, and N. Umino, "Quasi real-time fault model estimation for near-field tsunami forecasting based on RTK-GPS analysis: Application to the 2011 Tohoku-Oki earthquake (Mw 9.0)," Journal of Geophysical Research: Solid Earth, vol. 117, no. B2, 2012, b02311. [Online]. Available: http://dx.doi. org/10.1029/2011JB008750

[7] Y. Feng and J. Wang, "Exploring GNSS RTK Performance Benefits with GPS and Virtual Galileo Measurements," in Proceeding of the International Technical Meeting Institute of Navigation, 2007, pp. 218 2226.

[8] -, "GPS RTK performance characteristics and analysis," The Journal of Global Positioning Systems, vol. 7, no. 1, pp. 1-8, 2008. [Online]. Available: https://eprints.qut.edu. $\mathrm{au} / 30733 /$

[9] B. M. Scherzinger, "Precise Robust Positioning with Inertial/GPS RTK," 2000.

[10] "Precise Robust Positioning with Inertially Aided RTK,"

Navigation, vol. 53, no. 2, pp. 73-83, 2006. [Online]. Available: http://dx.doi. org/10.1002/j.2161-4296.2006.tb00374.x

[11] T. N. Thanh, N. C. Nghiem, T. T. Hung, and N. C. Ngon, "Evaluation of Real-Time Kinematic Positioning with Low-Cost, Single-Frequency GPS/GLONASS Receivers," in Proceedings of the Publishing House for Science and Technology, 2016.

[12] B. Matias, H. Oliveira, J. Almeida, A. Dias, H. Ferreira, A. Martins, and E. Silva, "High-accuracy low-cost RTK-GPS for an unmannned surface vehicle," in OCEANS 2015 Genova, May 2015, pp. 1-4.

[13] M. Skoglund, T. Petig, B. Vedder, H. Eriksson, and E. M. Schiller, "Static and dynamic performance evaluation of low-cost RTK GPS recei $\neg$ vers," in Proceedings of the 2016 IEEE Intelligent Vehicles Symposium (IV), June 2016, pp. 16-19. 
[14] T. Takasu, N. Kubo, and A. Yasuda, "Development, evaluation and application of rtldib: A program library for rtk-gps," in Proceedings of the International Symposium on GPS/GNSS, November 2007, pp. 213-218. 